\title{
GROUPS PRESENTED BY FINITE TWO-MONADIC CHURCH-ROSSER THUE SYSTEMS
}

\author{
J. AVENHAUS, K. MADLENER AND F. OTTO
}

\begin{abstract}
It is shown that a group $G$ can be defined by a monoid-presentation of the form $(\Sigma ; T)$, where $T$ is a finite two-monadic Church-Rosser Thue system over $\Sigma$, if and only if $G$ is isomorphic to the free product of a finitely generated free group with a finite number of finite groups.
\end{abstract}

Introduction. In 1911 M. Dehn formulated three fundamental problems for groups given by presentations of the form $\langle\Sigma ; L\rangle$, where $\Sigma$ is some set of generators, $\bar{\Sigma}$ is a disjoint copy of $\Sigma$, and $L \subseteq(\Sigma \cup \bar{\Sigma})^{*}$ is a set of defining relators [12]. One of these problems is the word problem, which can be stated as follows: Let $\langle\Sigma ; L\rangle$ be a group presentation. Given a word $w \in(\Sigma \cup \bar{\Sigma})^{*}$ decide in a finite number of steps whether $w$ defines the identity of the group presented by $\langle\Sigma ; L\rangle$.

In $1955 \mathrm{~W}$. W. Boone and P. S. Novikov independently proved that the word problem for finitely presented groups is undecidable [8, 20]. Furthermore, each sufficiently rich complexity class can be realized by the word problem for a finitely presented group $[\mathbf{1}, \mathbf{1 0}]$. However, since the property of having a $\mathbb{E}$-decidable word problem for some complexity class $\mathbb{E}$ is a Markov property, it is undecidable in general whether the group given by a presentation $\langle\Sigma ; L\rangle$ has a $\sqrt{ }$-decidable word problem [16]. In fact, it even is undecidable in general whether the group presented by $\langle\Sigma ; L\rangle$ is trivial, i.e., whether it is isomorphic to the trivial group $\langle 1\rangle$ [22]. However, if the given presentation is of a restricted form only, then it may give some information about the decidability and complexity of the word problem, e.g., if the presentation contains a single defining relator only, then the word problem is decidable [17], or if the presentation has the small cancellation property, then Dehn's linear time algorithm for deciding the word problem may be applied [16].

Recently there has been much interest in term rewriting systems because of their various applications to theorem proving, specification of abstract data types, program transformation and synthesis (see e.g. [9] for an overview). A restricted class of term rewriting systems are the so-called string rewriting systems, which actually are semi-Thue systems. It is hoped that by investigating these restricted systems one gets some insight into what can and what cannot be expected in the general situation [5].

Received by the editors March 5, 1985.

1980 Mathematics Subject Classification. Primary 20F10, 20F15; Secondary 02F05.

Key words and phrases. Group, monoid-presentation, Church-Rosser property, monadic system, context-free group, free product of groups. 
On the other hand, string rewriting systems have been used very successfully to provide efficient algorithms for some decision problems for monoids and groups [4-7, 21]. For example, if a group $G$ can be presented by a finite Church-Rosser Thue system, then the word problem for $G$ is decidable in linear time [4].

The classification of groups according to the complexity of their word problem and the characterization of groups whose word problem may be solved by algorithms of specific types have been studied extensively. Nevertheless there are only few results of algebraic characterizations of groups whose word problems are of certain complexities. One important result of this type is due to Muller and Schupp [19]: Groups with context-free word problems are essentially the virtually free groups, i.e., groups which have free subgroups of finite index. A subclass of these groups, the ones with simple context-free word problems, has been characterized by Haring-Smith [13].

Characterizations of groups that can be presented by rewriting systems satisfying certain restrictions are also of great interest because these groups have algorithms for solving the word problem which are directly derived from the presentations, and because these characterizations show the limitation of the method of using certain rewriting systems for solving word problems for groups. A very nice example of a result of this kind is due to Cochet. In [11] he shows that a group $G$ can be presented by a finite special Church-Rosser Thue system iff $G$ is isomorphic to a free product of finitely many cyclic groups.

In [2] Avenhaus and Madlener prove that a group $G$ can be presented by a finite monadic Church-Rosser Thue system that provides inverses of length one to all the generators if and only if $G$ is isomorphic to a free product of a finitely generated free group and finitely many finite groups. By coincidence this exactly is the class of groups for which the reduced word problem is a simple language [13].

In this paper the result of Avenhaus and Madlener mentioned above is extended to all finite monadic Church-Rosser Thue systems that have rules with left-hand side of length two only. Since every Thue system can be reduced effectively, and since the reduced forms of the Thue systems considered in [2] do have rules with left-hand sides of length two only, the result given here is a real extension of the one given in [2]. It is proved by reducing it to the one of [2], which is presented in $\$ 2$ to make this paper self-contained.

If $T$ is a finite monadic Church-Rosser Thue system over $\Sigma$, then each congruence class $[u]_{T}$ is a deterministic context-free language [4]. Hence, if a group $G$ can be described by a finite presentation involving a monadic Church-Rosser Thue system, then $G$ is a context-free group in the sense of Muller and Schupp [19]. Thus, the class of groups considered in this paper is a subclass of the class of context-free groups.

1. Definitions and notations. An alphabet $\Sigma$ is a finite set whose members are called letters. The set of words over $\Sigma$ is denoted by $\Sigma^{*}$, and 1 denotes the empty word. In general, $|x|$ denotes the length of a word $x$, which is defined by $|1|=0$, $|x a|=|x|+1$ for all $x \in \Sigma^{*}, a \in \Sigma$. For $a \in \Sigma,|w|_{a}$ denotes the number of 
occurrences of the letter $a$ in $w$. The identity of words is written as $\equiv$, and the concatenation of words $u$ and $v$ is simply written as $u v$.

A Thue system $T$ over $\Sigma$ is a (not necessarily finite) subset of $\Sigma^{*} \times \Sigma^{*}$. The members of $T$ are called (rewriting) rules. Given such a Thue system $T$ over $\Sigma$, domain $(T)=\left\{l \mid \exists r \in \Sigma^{*}:(l, r) \in T\right\}$, and range $(T)=\left\{r \mid \exists l \in \Sigma^{*}:(l, r) \in T\right\}$. A Thue system $T$ is called special if domain $(T) \subseteq \Sigma^{*}-\{1\}$, and range $(T)=\{1\}$, and it is called monadic if all its rules are length-reducing, i.e., $|l|>|r|$ for all $(l, r) \in T$, and range $(T) \subseteq \Sigma \cup\{1\}$. Finally, it is called two-monadic, if it is monadic, and domain $(T) \subseteq \Sigma^{2}$.

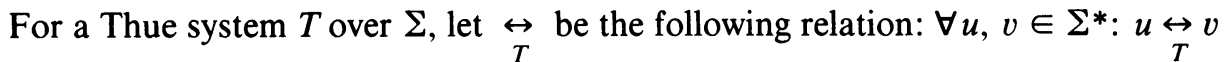
iff $\exists x, y \in \Sigma^{*},(l, r) \in T:(u \equiv x l y$ and $v \equiv x r y)$ or $(u \equiv x r y$ and $v \equiv x l y)$. The reflexive and transitive closure $\underset{T}{\leftrightarrow}$ of $\stackrel{\leftrightarrow}{T}$ is a congruence on $\Sigma^{*}$, the Thue congruence generated by $T$. If $\underset{T}{u \stackrel{*}{\leftrightarrow} v}$ one says that $u$ and $v$ are congruent (modulo $T)$. The congruence class $[u]_{T}$ of $u$ is the set $\left\{v \in \Sigma^{*} \mid \underset{T}{\stackrel{*}{\leftrightarrow}} v\right\}$.

Proposition 1.1 [15]. Let $T$ be a Thue system over $\Sigma$. Then the set of congruence classes $\left\{[u]_{T} \mid u \in \Sigma^{*}\right\}$ forms a monoid under the operation $[u]_{T}^{\circ} \circ[v]_{T}=[u v]_{T}$ with identity $[1]_{T}$. This monoid is denoted as $\Sigma^{*} / \stackrel{*}{\leftrightarrow}$.

Let $M$ be a monoid. If $M \cong \Sigma^{*} / \stackrel{*}{\leftrightarrow}$, i.e., if the monoids $M$ and $\Sigma^{*} / \stackrel{*}{\rightarrow}$ are isomorphic, then $(\Sigma ; T)$ is called a (monoid) presentation of $M, \Sigma$ is the set of generators, and $T$ is the set of defining relations of this presentation.

Let $T$ be a Thue system over $\Sigma$. Suppose $u, v \in \Sigma^{*}$, and $\underset{T}{u \leftrightarrow} v$. We write $\underset{T}{u} v$, if $|u|>|v|$. Then the Thue reduction defined by $T$ is the reflexive and transitive closure $\underset{T}{\vec{T}}$ of $\underset{T}{\rightarrow}$. Since words cannot have negative length, this relation is noetherian, i.e., there exists no infinite chain $u_{1} \rightarrow u_{2} \rightarrow u_{3} \rightarrow \vec{T} \cdots$ If $u \stackrel{*}{\rightarrow} v$, one says that $u$ reduces to $v, u$ is an ancestor of $v$, and $v$ is a descendant of $u$ (modulo $T$ ). If $u$ has no descendant except itself, then it is irreducible, otherwise it is reducible (modulo $T$ ). $\operatorname{IRR}(T)$ denotes the set of all irreducible words (modulo $T$ ).

Monadic Thue systems have the following nice property.

LEMma 1.2. Let $T$ be a monadic Thue system over $\Sigma$, and let $u, v, w \in \Sigma^{*}$ such that $u \underset{T}{\stackrel{*}{\rightarrow}} v w$. Then $u \equiv u_{1} u_{2}$, where $u_{1} \underset{T}{\stackrel{*}{\rightarrow}} v$, and $u_{2} \underset{T}{\stackrel{*}{\rightarrow}} w$.

Proof. We write $\underset{T}{\stackrel{k}{\rightarrow}} y$, if $x$ can be reduced to $y$ in $k$ steps. Now the lemma is proved by induction on $k$, where $u \stackrel{k}{\rightarrow} v w$.

$k=0$ : Then $u \equiv v w$, and nothing has to be shown.

$k \rightarrow k+1$ : Let $u \underset{T}{\stackrel{k+1}{\rightarrow}} v w$. Then there is some $z \in \Sigma^{*}$ such that $\underset{T}{\stackrel{k}{\rightarrow}} z$ and $z \rightarrow \vec{T} v w$. Hence, there are $x, \stackrel{T}{y} \in \Sigma^{*}$ and $(l, r) \in T$ with $z \equiv x l y$ and $v w^{T} \equiv x r y$. Now $T$ being monadic implies that $|r| \leqslant 1$, and therefore $r$ either belongs to $v$ or to $w$. Assume the first case, the other case being symmetric. Then $v \equiv x r y_{1}$, and $w \equiv y_{2}$ with $y \equiv y_{1} y_{2}$, and $z \equiv x l y_{1} y_{2}$. Thus, with $z_{1} \equiv x l y_{1}$, and $z_{2} \equiv y_{2}$ we have that 
$z \equiv z_{1} z_{2}, z_{1} \underset{T}{\rightarrow} v$, and $z_{2} \underset{T}{\stackrel{0}{\rightarrow}} w$. According to the induction hypothesis there exist $u_{1}$, $u_{2} \in \Sigma^{*}$ such that $u \equiv u_{1} u_{2}, u_{1} \underset{T}{\stackrel{*}{\rightarrow}} z_{1}$, and $u_{2} \underset{T}{\stackrel{*}{\rightarrow}} z_{2}$. Hence, $u \equiv u_{1} u_{2}, u_{1} \underset{T}{\stackrel{*}{\rightarrow}} v$, and $u_{2} \underset{T}{\stackrel{*}{\rightarrow}} w$.

It can be seen easily that in general Lemma 1.2 does not hold for nonmonadic Thue systems.

A Thue system $T$ over $\Sigma$ is Church-Rosser if every two congruent words have a

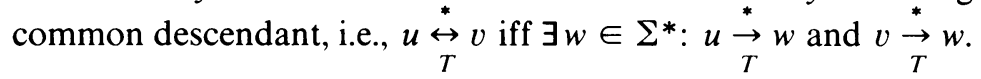

Proposition 1.3. The Thue system $T$ is Church-Rosser if and only if there exist no two distinct irreducible words that are congruent (modulo $T$ ).

Since every word is congruent to some irreducible word this says that in a Church-Rosser Thue system every congruence class contains exactly one irreducible word.

Proposition 1.4. [4]. If $T$ is a finite Thue system over $\Sigma$ that is Church-Rosser, then there exists a linear-time algorithm that on input a word $u \in \Sigma^{*}$ computes the irreducible descendant of $u$.

Hence, if $M=\Sigma^{*} / \stackrel{*}{\leftrightarrow}$ is a monoid, where $T$ is a finite Church-Rosser Thue system, then the word problem for $M$ is decidable in linear time, i.e., it is decidable in linear time whether two words $u, v \in \Sigma^{*}$ represent the same element of $M$.

Two Thue systems $T_{1}$ and $T_{2}$ over $\Sigma$ are equivalent, if the congruences $\stackrel{*}{\stackrel{*}{\leftrightarrow}}$ and $\stackrel{*}{\leftrightarrow}$ coincide, i.e., for all $u, v \in \Sigma^{*}, \underset{T_{1}}{\stackrel{*}{\leftrightarrow}} v$ if and only if $\underset{T_{2}}{\stackrel{*}{\leftrightarrow}} v$. A Thue system $T$ over $\Sigma$ is called reduced or normalized, if range $(T) \subseteq \operatorname{IRR}(T)$, and if $l \in \operatorname{IRR}(T-\{(l, r)\})$ for all rules $(l, r) \in T$.

Proposition $1.5[2,14]$. Let $T$ be a finite Church-Rosser Thue system over $\Sigma$. Then one can effectively compute a finite Church-Rosser Thue system $T^{\prime}$ over $\Sigma$ that is reduced, and that is equivalent to $T$.

In particular, if the finite Church-Rosser Thue system $T$ is special or monadic, then the reduced Thue system $T^{\prime}$ is also special or monadic, respectively. Further, if $M \cong \Sigma^{*} / \stackrel{\leftrightarrow}{\leftrightarrow}$, where $T$ is Church-Rosser and reduced containing a rule $(a, 1)$ for some $a \in \Sigma^{T}$, then $M$ is also described by the presentation $(\Sigma-\{a\} ; T-\{(a, 1)\})$. Thus we may assume that a reduced Church-Rosser Thue system does not contain rules of the form $(a, 1)$ with $a \in \Sigma$, i.e., $T \cap(\Sigma \times\{1\})=\varnothing$.

Proposition 1.6. Let $M=\Sigma^{*} / \stackrel{*}{\leftrightarrow}$. Then the monoid $M$ is actually a group iff every letter $a \in \Sigma$ is invertible in $M$, i.e., there exist words $u_{a}, v_{a} \in \Sigma^{*}$ with $a u_{a} \stackrel{*}{\leftrightarrow} 1 \stackrel{*}{\leftrightarrow} v_{T} a$.

In particular, $a u_{a} \stackrel{*}{\leftrightarrow} 1 \stackrel{*}{\leftrightarrow} v_{a} a$ imply that $u_{a} \stackrel{*}{\leftrightarrow} v_{a}$. Further, if $M=\Sigma^{*} / \stackrel{*}{\leftrightarrow}$ happens to be a group, then every cyclic permutation of a word representing the identity 
also represents the identity, i.e., $u v \underset{T}{\stackrel{*}{\leftrightarrow}} 1$ implies that $v u \stackrel{*}{\leftrightarrow} \underset{T}{1}$, too. So when $T$ is also Church-Rosser, then with $u v \stackrel{*}{\rightarrow} 1$ also $v u \stackrel{*}{\rightarrow} 1$.

Finitely generated free groups and finite cyclic groups can be presented by finite special Church-Rosser Thue systems, namely $\left(\Sigma_{+} \cup \Sigma_{-} ;\left\{(a \bar{a}, 1),(\bar{a} a, 1) \mid a \in \Sigma_{+}\right\}\right)$ and $\left(\{a\} ;\left\{\left(a^{n}, 1\right)\right\}\right)$ represent the free group in the generators $\Sigma$ and the cyclic group of order $n$, respectively. Here $\Sigma_{-}$is an alphabet in 1-1 correspondence with $\Sigma_{+}$, and the function ${ }^{-}: \Sigma_{+} \rightarrow \Sigma_{-}$is realizing this correspondence. A presentation for the free product of two groups is obtained by taking as generators the disjoint union of the generators and as Thue system the disjoint union of the Thue systems representing each group. So the free product of groups presented by finite special Church-Rosser Thue systems has itself such a presentation. A characterization of all groups presented by such systems is given by Cochet in [11].

THEOREM 1.7 [11]. A group $G$ can be presented by a presentation of the form $(\Sigma ; T)$, where $T$ is a finite, special, Church-Rosser Thue system iff $G$ is the free product of finitely many cyclic groups.

2. Thue systems all the generators of which have inverses of length one. Here we want to characterize the groups that can be presented by presentations of the form ( $\Sigma ; T)$, where $T$ is a finite monadic Church-Rosser Thue system such that for every $a \in \Sigma$ some $b \in \Sigma$ exists with $a b \underset{T}{\rightarrow} 1$, i.e., all the generators in the given presentation $(\Sigma ; T)$ have inverses of length one.

Since the reduced form of a monadic Church-Rosser Thue system is itself monadic and Church-Rosser, we can restrict our attention to reduced Thue systems.

LEMMA 2.1. Let $T$ be a finite monadic Church-Rosser Thue system over $\Sigma$ such that every element of $\Sigma$ has an inverse of length one with respect to $T$. If $T$ is reduced, then $T \subseteq \Sigma^{2} \times(\Sigma \cup\{1\})$

Proof. Since $T$ is reduced and monadic, we have $|l| \geqslant 2$ for every $l \in$ domain $(T)$. Now let $(l, r) \in T$ with $l \equiv a z$ for some $a \in \Sigma$. Since every element of $\Sigma$ has an inverse of length one with respect to $T$, there is some $b \in \Sigma$ with $a b \underset{T}{\stackrel{*}{\rightarrow}} 1$, and $b a \stackrel{*}{\rightarrow}$ 1. In particular, $(\Sigma ; T)$ is a group, and therefore $b r \underset{T}{\stackrel{*}{\rightarrow}} z . T$ being reduced implies that $z$ is irreducible, and hence $b r \stackrel{*}{\rightarrow} z$. Thus, $|b r|=1+|r| \geqslant|z|$.

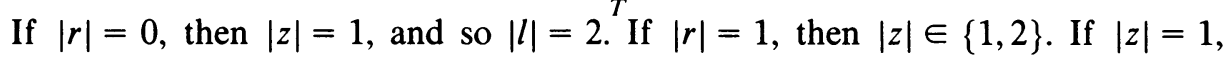
then $|l|=2$, and if $|z|=2$, then $z \equiv b r$ implying $l \equiv a z \equiv a b r$. However, this contradicts the assumption of $T$ being reduced, since $a b \stackrel{*}{\rightarrow} 1$.

So the presentations considered here are of a very special form.

If a group $G$ is finite, then its multiplication table is finite. So we get a monadic presentation for $G$ by taking for each element $(\neq 1)$ of the group a letter and for $T=\{(a b, c) \mid a \cdot b=c\}$. It is easy to see that this is a finite reduced monadic Church-Rosser presentation for $G$. 
So if 'a group $G$ is finite or free, then $G$ has a presentation of this special form. If $G_{1}$ and $G_{2}$ have such presentations, then the free product $G_{1} * G_{2}$ does so as well. We will show that no other groups have such presentations, and by doing so we will prove

THEOREM 2.2. A group $G$ can be presented by a presentation of the form $(\Sigma ; T)$, where $T$ is a finite, monadic, Church-Rosser Thue system such that every element of $\Sigma$ has an inverse of length one with respect to $T$ iff $G$ is a free product of a finitely generated free group and finitely many finite groups.

We will use a result of Haring-Smith [13] that is based on the work of Stallings [23], and that gives a geometrical (language) characterization of groups which are free products of free groups with finite groups. This characterization is based on the Cayley diagram of a presentation of the group by generators and defining relations.

Suppose that $\Sigma=\Sigma_{+} \cup \Sigma_{-}$, where $\Sigma_{-}=\left\{\bar{a} \mid a \in \Sigma_{+}\right\}, \Sigma_{+} \cap \Sigma_{-}=\varnothing$, and let $R \subseteq \Sigma^{*} \times \Sigma^{*}$ be finite. Then the monoid $\Sigma^{*} / \underset{T}{\leftrightarrow}$ with $T=R \cup\{(a \bar{a}, 1),(\bar{a} a, 1)\}$ $\left.a \in \Sigma_{+}\right\}$is a group, and $\left\langle\Sigma_{+} ; R\right\rangle$ is a group presentation of this group [18].

THEOREM 2.3 [13]. Let $\left\langle\Sigma_{+} ; R\right\rangle$ be a group presentation of a group $G$ such that the set $M_{0}=\left\{w \in\left(\Sigma_{+} \cup \Sigma_{-}\right)^{*} \mid w=1\right.$ in $G$, but no proper segment of $w$ is equal to 1 in $G\}$ is finite. Then $\dot{G}$ is a free product of a finitely generated free group with finitely many finite groups.

We will use this theorem for our characterization by proving

Lemma 2.4. Given a finite monadic Church-Rosser Thue system $T$ over $\Sigma$ such that every element of $\Sigma$ has an inverse of length one with respect to $T$. Then the following set $M$ is finite: $M=\left\{w \in \Sigma^{*} \mid w \underset{T}{\stackrel{*}{\leftrightarrow}} 1\right.$, but no proper segment $u$ of $w$ satisfies $\left.\underset{T}{u} \stackrel{*}{\leftrightarrow} 1\right\}$.

Proof. Without loss of generality we may assume that $T$ is reduced. For each generator $a \in \Sigma$ denote by $\bar{a}$ the inverse of length one of $a$.

Now we proceed by showing that each $w \in \Sigma^{*}$ with $\underset{T}{w \stackrel{*}{\leftrightarrow}} 1$ contains a proper segment $v$ satisfying $v \stackrel{*}{\leftrightarrow} 1$, if $|w|>n:=|\Sigma|+2$. Here $|\Sigma|$ denotes the cardinality of $\Sigma$.

Suppose that $w \equiv a_{1} \cdots a_{n} \stackrel{*}{\leftrightarrow} 1$, where $a_{1}, \ldots, a_{n} \in \Sigma$, and let $u_{i}$ denote the irreducible descendant of the initial factor $a_{1} \cdots a_{i}$ of $w$, i.e., $a_{1} \cdots a_{i} \stackrel{*}{T} u_{i}$ $(1 \leqslant i \leqslant n)$. If $\left|u_{i}\right| \leqslant 1$ for all $1 \leqslant i \leqslant n$, then there are $i$ and $j, 1 \leqslant i<j \leqslant n$, with $u_{i} \equiv u_{j}$. Hence,

$$
a_{1} \cdots a_{i} \stackrel{*}{T} u_{i} \equiv u_{j} \stackrel{*}{T} a_{1} \cdots a_{i} a_{i+1} \cdots a_{j}
$$

and so $v \equiv a_{i+1} \cdots a_{j} \stackrel{*}{\leftrightarrow} 1$, since $\Sigma^{*} / \stackrel{*}{\leftrightarrow}$ is a group.

Now assume there is some $u_{i}$ with $\left|u_{i}\right| \geqslant 2$. Since $u_{n-1} \equiv \bar{a}_{n}$, this implies that $\left|u_{j+1}\right|<\left|u_{j}\right|$ for some $j<n-1$. Of course, $u_{j} \stackrel{*}{\leftrightarrow} u_{j+1} \bar{a}_{j+1}$, and so $u_{j+1} \bar{a}_{j+1} \stackrel{*}{\rightarrow} u_{j}$, 
since $u_{j}$ is irreducible. Hence, $\left|u_{j}\right| \leqslant\left|u_{j+1}\right|_{*}+1<\left|u_{j}\right|+1$. Therefore, $\left|u_{j}\right|=\left|u_{j+1}\right|$ +1 , and $u_{j} \equiv u_{j+1} \bar{a}_{j+1}$. Thus, $a_{1} \cdots a_{j} \underset{T}{\rightarrow} u_{j} \equiv u_{j+1} \bar{a}_{j+1}$, and so there is some $k, 1 \leqslant k<j$, such that $a_{1} \cdots a_{k} \underset{T}{\stackrel{*}{\rightarrow}} u_{j+1}$, and $a_{k+1} \cdots a_{j} \stackrel{*}{\rightarrow} \bar{a}_{j+1}$. This follows from Lemma 1.2. Hence, $w$ contains the segment $a_{k+1} \cdots a_{j} a_{j+1} \stackrel{*}{\rightarrow} \bar{a}_{j+1} a_{j+1} \underset{T}{\rightarrow} 1$.

Notice that Lemma 2.4 also holds for finite special Church-Rosser Thue systems $T$ without the assumption on the inverses of length one.

Now we can prove Theorem 2.2. Suppose that a group $G$ is presented by a presentation of the form $(\Sigma ; T)$, where $T$ is a finite, monadic, Church-Rosser Thue system such that every element of $\Sigma$ has an inverse of length one with respect to $T$. If we take $\Sigma_{+}:=\Sigma$ and $R:=T$, then $G$ is isomorphic to the group $G_{0}$ presented by $\left\langle\Sigma_{+} ; R\right\rangle$. According to Lemma 2.4 the set $M=\left\{w \in \Sigma^{*} \mid w \underset{T}{\leftrightarrow} 1\right.$, but no proper segment $u$ of $w$ satisfies $\underset{T}{u} \stackrel{*}{\leftrightarrow} 1\}$ is finite. Since in $(\Sigma ; T)$ each generator has an inverse of length one, it is easy to see that with $M$ also the set

$$
M_{0}=\left\{w \in\left(\Sigma_{+} \cup \Sigma_{-}\right)^{*} \mid w=1 \text { in } G_{0},\right.
$$

\section{but no proper segment of $w$ is equal to 1 in $\left.G_{0}\right\}$}

is finite. Thus, Theorem 2.3 gives the intended result.

In the next section we will omit the condition that each generator in the presentation $(\Sigma ; T)$ under consideration has an inverse of length one. However, in order to be able to prove the intended characterization, we have to restrict our attention to Thue systems all the rules of which have left-hand sides of length two.

3. Two-monadic Thue systems. If a finite monadic Church-Rosser Thue system $T$ over $\Sigma$ is reduced, and if each generator $a \in \Sigma$ has an inverse of length one, i.e., if for each $a \in \Sigma$ there is some $b \in \Sigma$ such that $a b \stackrel{*}{\leftrightarrow} 1$, then the left-hand side of each rule of $T$ is of length two, i.e., $T \subseteq \Sigma^{2} \times(\Sigma \cup\{1\})$. On the other hand, a finite Church-Rosser Thue system $T \subseteq \Sigma^{2} \times(\Sigma \cup\{1\})$ can be reduced without having an inverse of length one for each of the generators from $\Sigma$, although $\Sigma^{*} / \underset{T}{\leftrightarrow}$ is a group.

EXAMPLE 3.1. Take $\Sigma=\{a, b, c\}$, and $T=\left\{\left(a^{2}, 1\right),\left(b^{2}, 1\right)\right.$, $(a b, c),(a c, b),(c b, a)\}$. Then $T$ is reduced, and $T \subseteq \Sigma^{2} \times(\Sigma \cup\{1\})$. It can be checked easily that $T$ is Church-Rosser.

It is obvious that the generator $c$ does not have an inverse of length one. However, $\underset{c b}{a} \underset{T}{\rightarrow} a^{2} \underset{T}{\rightarrow}$, and $b a c_{\vec{T}} \underset{T}{b^{2}} \underset{T}{\rightarrow}$, i.e., $c$ is invertible, and hence, $\Sigma^{*} / \stackrel{*}{\leftrightarrow}$ is a group.

Theorem 2.2 was proved by showing that the theorem of Haring-Smith can be applied to a group-presentation $(\Sigma ; T)$, where $T$ is a finite monadic Church-Rosser Thue system that is reduced and that has inverses of length one for all the generators. Now one may hope that this theorem can be applied also in the more general situation, where $T \subseteq \Sigma^{2} \times(\Sigma \cup\{1\})$ does not have inverses of length one for all the generators. 
EXAmPle 3.1 (CONTINUED). $\quad c a c_{T} \underset{T}{\rightarrow} c b \underset{T}{\rightarrow} a$, and hence $c^{m} a c^{m} \stackrel{*}{\leftrightarrow} a$. Thus, $c^{m} a c^{m} a \stackrel{*}{\leftrightarrow} 1$. But no proper segment of $c^{m} a c^{m} a$ is congruent to 1 modulo $T$. Hence,

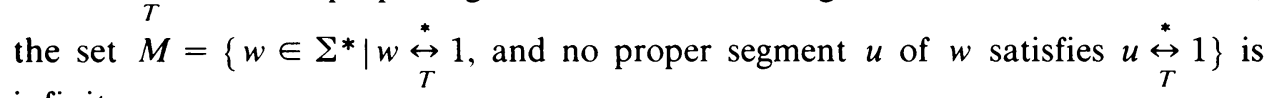
infinite.

Thus, the theorem of Haring-Smith cannot be applied to every presentation ( $\Sigma ; T)$ defining a group, where $T \subseteq \Sigma^{2} \times(\Sigma \cup\{1\})$ is finite, reduced, and ChurchRosser. To get around this difficulty one can try to introduce the missing inverses.

EXAMPle 3.1 (CONTINUED). Take $\Sigma_{0}=\Sigma \cup\{\bar{c}\}$. Since $\bar{c}$ is to act as the inverse of $c$, we need the rules $(c \bar{c}, 1)$ and $(\bar{c} c, 1)$. Take $T_{0}=T \cup\{(c \bar{c}, 1),(\bar{c} c, 1)\}$. Now assume that $\Sigma_{1}$ is a finite alphabet containing $\Sigma_{0}$, and $T_{*}$ is a finite Church-Rosser Thue system containing $T_{0}$ such that $\Sigma^{*} / \underset{T}{\stackrel{*}{\leftrightarrow}} \cong \Sigma_{1}^{*} / \stackrel{\stackrel{\leftrightarrow}{\leftrightarrow}}{T_{1}}$, and when restricted to $\Sigma^{*}$, the congruences $\underset{T}{\stackrel{*}{\leftrightarrow}}$ and $\underset{T_{1}}{\stackrel{*}{\leftrightarrow}}$ coincide. Since $b \underset{T_{0}}{\leftarrow \leftarrow} \bar{c} b \underset{T_{0}}{\rightarrow} \bar{c} a$, we have $\bar{c} a \underset{T_{1}}{\rightarrow} b$. Hence, $b a \underset{T_{1}}{\leftarrow} \bar{c} a^{2} \underset{T_{1}}{\rightarrow} \bar{c}$, and so $b a \underset{T_{1}}{\rightarrow} \bar{c}$. Notice that $\underset{T_{1}}{x \stackrel{*}{\leftrightarrow}} 1$ for all $x \in \Sigma_{0}$, since the congruences $\underset{T}{\stackrel{*}{\leftrightarrow}}$ and $\underset{T_{1}}{\stackrel{*}{\leftrightarrow}}$ coincide on $\Sigma^{*}$.

Now

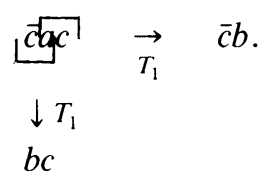

Hence, there is some $f \in \Sigma_{1} \cup\{1\}$ such that $(\bar{c} b, f),(b c, f) \in T_{1}$. Thus,

$$
\begin{array}{lll}
\sqrt[d b c]{ } \overrightarrow{T_{1}} & \text { af, } \\
\downarrow T_{1} & \\
c^{2} &
\end{array}
$$

and hence there is some $g \in \Sigma_{1} \cup\{1\}$ such that $\left(c^{2}, g\right) \in T_{1}$. Since $\Sigma_{1}^{*} / \stackrel{*}{\leftrightarrow} \vec{T}_{1}$ is a group, Lemma 4 of [3] implies that $c$ has finite order in $\Sigma_{1}^{*} / \stackrel{*}{\leftrightarrow} \underset{T_{1}}{\stackrel{*}{*}}$. On the other hand, $\Sigma_{1}^{*} / \stackrel{*}{\leftrightarrow}$ is isomorphic to the free product $G:=\left\langle a ; a^{2}\right\rangle *\left\langle b ; b^{2}\right\rangle$, and $\operatorname{ord}_{G}(c)=$ $\operatorname{ord}_{G}(a b)=0$, i.e., $c$ has infinite order $h$.

Thus, when $\bar{c}$ is added, then no monadic Church-Rosser Thue system presenting the group $\Sigma^{*} / \stackrel{\leftrightarrow}{\leftrightarrow}$ can be reached.

Another possible way is to try to get rid of all those generators that do not have inverses of length one. As we will see in the following this can actually be done for the presentation under consideration.

THEOREM 3.2. Let $T \subseteq \Sigma^{2} \times(\Sigma \cup\{1\})$ be a finite Church-Rosser Thue system such that $\Sigma^{*} / \underset{T}{\leftrightarrow}$ is a group. Then there exists a subset $\Sigma_{1}$ of $\Sigma$ such that the following conditions are satisfied:

(1) each letter from $\Sigma_{1}$ has an inverse of length 1 ;

(2) the Thue system $T_{1}:=T \cap\left(\Sigma_{1}^{2} \times\left(\Sigma_{1} \cup\{1\}\right)\right)$ is Church-Rosser; and

(3) $\left(\Sigma_{1} ; T_{1}\right)$ presents the same group as $(\Sigma ; T)$, i.e., $\Sigma_{1}^{*} / \underset{T_{1}}{\stackrel{*}{\leftrightarrow}} \cong \Sigma^{*} / \underset{T}{\stackrel{*}{\leftrightarrow}}$. 
Together with Theorem 2.2 this gives our main result.

THEOREM 3.3. A group $G$ has a presentation $(\Sigma ; T)$ such that $T \subseteq \Sigma^{2} \times(\Sigma \cup\{1\})$ is finite, and Church-Rosser iff $G$ is a free product of a finitely generated free group with finitely many finite groups.

It remains to prove Theorem 3.2. This proof is subdivided into several lemmata. In the following let $T \subseteq \Sigma^{2} \times(\Sigma \cup\{1\})$ denote a fixed finite Church-Rosser Thue system such that $G=\Sigma^{*} / \underset{T}{\stackrel{*}{\leftrightarrow}}$ is a group. Notice that $T$ is reduced.

Now each generator from $\Sigma$ can be classified according to the length of a corresponding irreducible inverse.

Definition 3.4. For $a \in \Sigma$ define $\lambda(a)=\min \{|u| \mid a u \underset{T}{\stackrel{*}{\rightarrow}} 1\}$. Let $\Sigma_{i}=\{a \in$ $\Sigma \mid \lambda(a)=i\}$.

Since $G$ is a group, we have $\Sigma=\bigcup_{i \geqslant 1} \Sigma_{i}$, where $\Sigma_{i} \cap \Sigma_{j}=\varnothing$ for $i \neq j$. In particular, $\Sigma_{1} \neq \varnothing$, since $T$ is two-monadic.

Lemma 3.5. Let $i \geqslant 1$, and let $a \in \Sigma_{i}$. Then the set $\Sigma^{i} \cap\{u \mid a u \underset{T}{\stackrel{*}{\rightarrow}} 1\}$ consists of $a$ single irreducible word $u_{a}$ from $\Sigma_{1}^{i}$.

Proof. Let $a \in \Sigma_{i}$ for some $i \geqslant 1$. Then according to the definition of $\Sigma_{i}$, $\Sigma^{i} \cap\{u \mid a u \underset{T}{\stackrel{*}{\rightarrow}} 1\} \subseteq \operatorname{IRR}(T)$, i.e., all the words in $\Sigma^{i} \cap\{u \mid a u \stackrel{*}{\rightarrow} 1\}$ are irreducible.

Now let $u_{1}^{T}, u_{2} \in \Sigma^{i} \cap\{u \mid a u \underset{T}{\stackrel{*}{\rightarrow}} 1\}$. Then $a u_{1} \underset{T}{\stackrel{*}{\rightarrow}} 1 \underset{T}{\stackrel{*}{\leftarrow}} a u_{2} . \stackrel{T}{T}$ But $G$ is a group, and hence the equation $a u_{1} \stackrel{*}{\leftrightarrow} a u_{2}$ implies $u_{1} \stackrel{*}{\leftrightarrow} u_{2}$. Since $T$ is Church-Rosser, and since $u_{1}$ and $u_{2}$ are both irreducible, this means $u_{1} \equiv u_{2}$. Thus, $\Sigma^{i} \cap\{u \mid a u \stackrel{*}{\rightarrow} 1\}$ consists of a single irreducible word $u_{a}$. It remains to prove that $u_{a} \in \Sigma_{1}^{*}$. This is done by induction on $i$.

$i=1$ : Let $a \in \Sigma_{1}$. Then $u_{a} \in \Sigma^{1}$. Now $a u_{a} \underset{T}{\stackrel{*}{\rightarrow}} 1$, and since $G$ is a group, $u_{a} a \stackrel{*}{\rightarrow} 1$. Hence $u_{a} \in \Sigma_{1}^{1}$.

$i \rightarrow i+1$ : Let $a \in \Sigma_{i+1}$. Then $u_{a} \equiv b_{i+1} b_{i} \cdots b_{1} \in \Sigma^{i+1}$. Since $u_{a}$ is irreducible, there is some $c \in \Sigma$ such that $\left(a b_{i+1}, c\right) \in T$ and $c b_{i} b_{i-1} \cdots b_{1} \underset{T}{\stackrel{*}{\rightarrow}} 1$. Hence $c \in \Sigma_{i}$, and $u_{c} \equiv b_{i} b_{i-1} \cdots b_{1}$. According to the induction assumption, $u_{c} \in \Sigma_{1}^{i}$, i.e., $b_{1}, b_{2}, \ldots, b_{i} \in \Sigma_{1}$.

$G$ is a group, and so $a u_{a} \underset{T}{\stackrel{*}{\rightarrow}} 1$ implies $u_{a} \underset{T}{\stackrel{*}{\rightarrow}}$. Thus, there is some $d \in \Sigma$ such that $\left(b_{1} a, d\right) \in T$ and $b_{i+1} b_{i} \cdots b_{2} \underset{T}{\stackrel{*}{\rightarrow}} 1$. Hence, $d \in \Sigma_{i}$, and $u_{d} \equiv b_{i+1} b_{i} \cdots b_{2}$. Again the induction assumption can be applied giving $u_{d} \in \Sigma_{1}^{i}$, i.e., $b_{2}, \ldots, b_{i}, b_{i+1}$ $\in \Sigma_{1}$. Hence, $u_{a} \equiv b_{i+1} b_{i} \cdots b_{2} b_{1} \in \Sigma_{1}^{i+1}$.

From the proof of Lemma 3.5 we see immediately that, if $\Sigma_{i+1} \neq \varnothing$, then $\Sigma_{\mathrm{i}} \neq \varnothing$ as well. Hence, $\Sigma=\bigcup_{i=1}^{m} \Sigma_{i}$, where $m \leqslant|\Sigma|$. Further, Lemma 3.5 implies the following

Corollary 3.6. Let $i \geqslant 1$, and let $a \in \Sigma_{i}$. Then the set $\Sigma_{1}^{i} \cap\{v \mid v \stackrel{*}{\leftrightarrow} a\}$ consists of a single word $v_{a}$. 
Proof. Let $i \geqslant 1$, and let $a \in \Sigma_{i}$. Then there is a word $u_{a} \in \Sigma_{1}^{i}$ with $a u_{a} \stackrel{*}{\rightarrow} 1$. Let $u_{a} \equiv b_{1} \cdots b_{i}$. Then for each letter $b_{j}$, there is some letter $\bar{b}_{i} \in \Sigma_{1}$ such that $\bar{b}_{j} b_{j} \underset{T}{\rightarrow}$. Take $v_{a} \equiv \bar{b}_{i} \cdots \bar{b}_{1}$. Then $v_{a} u_{a} \equiv \bar{b}_{i} \cdots \bar{b}_{1} b_{1} \cdots b_{i} \underset{T}{\stackrel{*}{\rightarrow}} 1 \underset{T}{\stackrel{*}{\leftarrow}} a u_{a}$. Since $G$ is a group, this implies $v_{a} \stackrel{*}{\leftrightarrow} a$, i.e., $v_{a} \in \Sigma_{1}^{i} \cap\{v \mid v \stackrel{*}{\leftrightarrow} a\}$.

Now let $u \in \Sigma_{1}^{i} \cap\{v \mid v \stackrel{*}{\leftrightarrow} a\}$, i.e., $u \equiv c_{i} \cdots c_{1}$ with $c_{1}, \ldots, c_{i} \in \Sigma_{1}$ such that $u \stackrel{*}{\leftrightarrow} a$. For each letter $c_{j}$, there is some letter $\bar{c}_{j} \in \Sigma_{1}$ such that $c_{j} \bar{c}_{j} \rightarrow 1$. Take $\hat{u} \stackrel{T}{\equiv} \bar{c}_{1} \ldots \bar{c}_{i}$. Then

$$
a \hat{u} \stackrel{*}{\leftrightarrow} u \hat{u} \equiv c_{i} \cdots c_{1} \bar{c}_{1} \cdots \bar{c}_{i} \underset{T}{\stackrel{*}{\rightarrow}} 1 \underset{T}{\stackrel{*}{\leftarrow}} a u_{a},
$$

and hence, $\hat{u} \stackrel{*}{\leftrightarrow} u_{a}$. But $|\hat{u}|=i=\left|u_{a}\right|$, and $u_{a}$ being irreducible imply that $\hat{u} \equiv u_{a}$, i.e., $b_{j} \equiv \bar{c}_{j}$ for $j=1, \ldots, i$. Hence, Lemma 3.5 gives $\bar{b}_{j} \equiv c_{j}$ for $j=1, \ldots, i$, i.e., $u \equiv c_{i} \cdots c_{1} \equiv \bar{b}_{i} \cdots \bar{b}_{1} \equiv v_{a}$. Thus, $\Sigma_{1}^{i} \cap\{v \mid v \stackrel{*}{\leftrightarrow} a\}=\left\{v_{a}\right\}$.

Thus, the group $G$ is generated by $\Sigma_{1}$ already. For each letter $a \in \Sigma_{1}$, denote the word $u_{a} \in \Sigma_{1}$ by $\bar{a}$, and for $w \equiv a_{1} \cdots a_{k} \in \Sigma_{1}^{k}$, denote the word $\bar{a}_{k} \cdots \bar{a}_{1}$ by $w^{-1}$. Notice that for every $a \in \Sigma_{1}$ the rules $(a \bar{a}, 1)$ and $(\bar{a} a, 1)$ are in $T$.

Since we want to get rid of the generators from $\Sigma-\Sigma_{1}$, we define a mapping $\varphi$ : $\Sigma \rightarrow \Sigma_{1}^{*}$ and a subsystem $T_{1}$ of $T$ as follows.

DEFINITION 3.7. (i) For $a \in \Sigma_{i}$ let $\varphi(a) \equiv v_{a} \in \Sigma_{1}^{i}$.

(ii) Take $T_{1}=T \cap\left(\Sigma_{1}^{2} \times\left(\Sigma_{1} \cup\{1\}\right)\right)$, i.e., $T_{1}=\left\{(l, r) \in T \mid l, r \in \Sigma_{1}^{*}\right\}$.

Because of Corollary 3.6 the mapping $\varphi$ is well defined. It can be extended to $\Sigma^{*}$ in an obvious way.

Now we want to show that $\left(\Sigma_{1} ; T_{1}\right)$ is a presentation of the group $G$ such that Theorem 2.2 applies to this presentation. However, before we can start with doing so, we need to derive one further property of the system $T$.

LEMMA 3.8. Let $c \in \Sigma_{i}$ for some $i \geqslant 2$, and let $a_{1}, a_{2}, b_{1}, b_{2} \in \Sigma_{1}$ with $\left(a_{1} a_{2}, c\right)$, $\left(b_{1} b_{2}, c\right) \in T$. Then $a_{1} a_{2} \equiv b_{1} b_{2}$.

Proof. We have $a_{1} a_{2} \underset{T}{c} \underset{T}{\leftarrow} b_{1} b_{2}$. Hence

$$
c \bar{b}_{2} \bar{b}_{1} \stackrel{\leftrightarrow}{\leftrightarrow} b_{1}^{T} b_{2} \bar{b}_{2} \bar{b}_{1} \underset{T}{\stackrel{*}{\rightarrow}} 1 \stackrel{*}{\leftarrow} a_{1} a_{2} \bar{a}_{2} \bar{a}_{1} \underset{T}{\stackrel{\leftrightarrow}{\leftrightarrow}} c \bar{a}_{2} \bar{a}_{1} .
$$

Since $c \in \Sigma_{i}$ for some $i \geqslant 2$, this implies that $i=2$, and $\bar{b}_{2} \bar{b}_{1} \equiv c^{-1} \equiv \bar{a}_{2} \bar{a}_{1}$. Hence by Lemma 3.5, $a_{1} a_{2} \equiv b_{1} b_{2}$.

Now we are ready to prove

LEMMA 3.9. $T_{1}$ is finite, monadic, and Church-Rosser.

Proof. $T_{1}$ is finite and monadic, since it is a subsystem of $T$. It remains to prove that $T_{1}$ is Church-Rosser. For doing so, we have to check all the critical pairs of $T_{1}$. So assume that $(a b, d)$. $(b c, f) \in T_{1}$ with $a, b, c \in \Sigma_{1}$, and $d, f \in \Sigma_{1} \cup\{1\}$. This gives the critical pair ( $d c$, af ): $d c \leftarrow a b c \rightarrow$ af.

If $d \equiv 1$ and $f \equiv 1$, then $a \equiv \bar{b}$ and $c \equiv \bar{b}$. Hence, $d c \equiv \bar{b} \equiv a f$.

If $d \equiv 1$ and $f \in \Sigma_{1}$, then $d c \equiv c \underset{T_{1}}{\stackrel{*}{\leftrightarrow}}$ af. But $T_{1} \subseteq T$ implying $c \underset{T}{\stackrel{*}{\leftrightarrow}}$ af. Since $T$ is Church-Rosser, this means $(a f, c) \in T$, and so $(a f, c) \in T_{1}$. 
If $d \in \Sigma_{1}$ and $f \equiv 1$, then $(d c, a) \in T_{1}$ for similar reasons.

So assume $d \in \Sigma_{1}$ and $f \in \Sigma_{1}$. Now $d c \stackrel{*}{\leftrightarrow}$ af implies that $d c \underset{T}{\stackrel{*}{\leftrightarrow}}$ af, and since $T$ is Church-Rosser, there is some $g \in \Sigma \cup\{1\}$ such that $(d c, g) \in T$ and $(a f, g) \in T$. If $g \in \Sigma_{1} \cup\{1\}$, then $(d c, g) \in T_{1}$ and $(a f, g) \in T_{1}$. If $g \in \Sigma_{i}$ for some $i \geqslant 2$, then Lemma 3.8 applies giving $d c \equiv a f$.

Thus, in every case the critical pair $(d c, a f)$ can be resolved in $T_{1}$. Hence, $T_{1}$ is Church-Rosser.

Obviously, in the monoid $\Sigma_{1}^{*} / \underset{T_{1}}{\stackrel{*}{\leftrightarrow}}$ every generator has an inverse of length one. In particular, $\Sigma_{1}^{*} / \underset{T_{1}}{\stackrel{*}{\leftrightarrow}}$ is a group, and therefore Theorem 2.2 applies to this presentation. Hence, we have

COROLLARY 3.10. The group $\Sigma_{1}^{*} / \underset{T_{1}}{\stackrel{*}{\leftrightarrow}}$ is a free product of a finitely generated free group with finitely many finite groups.

In order to prove Theorem 3.2 it only remains to show that the mapping $\varphi$ defines an isomorphism from $\Sigma^{*} / \underset{T}{\stackrel{*}{\leftrightarrow}}$ onto $\Sigma_{1}^{*} / \underset{T_{1}}{\stackrel{*}{\leftrightarrow}}$. Obviously, $\varphi$ is onto, since $\varphi(a) \equiv a$ for all $a \in \Sigma_{1}$. Further, if $\varphi(w) \underset{T_{1}}{\stackrel{*}{\leftrightarrow}} 1$ for some $w \in \Sigma^{*}$, then $\varphi(w) \underset{T}{\stackrel{*}{\leftrightarrow}} 1$, since $T_{1} \subseteq T$. But $\varphi(w) \stackrel{\leftrightarrow}{\stackrel{*}{\leftrightarrow}} w$ according to the definition of $\varphi$, and so $\underset{T}{\stackrel{*}{\leftrightarrow}} 1$. Thus, if $\varphi$ is indeed a homomorphism from $\Sigma^{*} / \underset{T}{\stackrel{*}{\leftrightarrow}}$ in $\Sigma_{1}^{*} / \underset{T_{1}}{\stackrel{*}{\leftrightarrow}}$, then $\varphi$ is an isomorphism. Therefore, we have to show that $\varphi(a b) \underset{T_{1}}{\stackrel{*}{\leftrightarrow}} \varphi(c)$ for all rules $(a b, c) \in T$. For this we need one further lemma.

Lemma 3.11. Let $u, v \in \Sigma_{1}^{*}$ with $u \underset{T_{1}}{\stackrel{k}{\rightarrow}} v$. Then also $u^{-1} \underset{T_{1}}{\stackrel{k}{\rightarrow}} v^{-1}$.

Proof. Let $u \equiv a_{1} \cdots a_{n}$, and $v \equiv b_{1} \cdots b_{m}$ with $a_{i}, b_{j} \in \Sigma_{1}$. Then $u^{-1} \equiv \bar{a}_{n}$ $\cdots \bar{a}_{1}$, and $v^{-1} \equiv \bar{b}_{m} \cdots \bar{b}_{1}$. Now we proceed by induction on $k$.

$k=0: u \underset{T_{1}}{\rightarrow} v$, i.e., $u \equiv v$. Hence, $u^{-1} \equiv v^{-1}$, and so $u^{-1} \underset{T_{1}}{\stackrel{0}{\rightarrow}} v^{-1}$.

$k=1: u \stackrel{1}{\rightarrow} v$, i.e., $n \in\{m+1, m+2\}$.

If $n=m+2$, then there is some $i \in\{1, \ldots, n-1\}$ such that $a_{i+1} \equiv \bar{a}_{i}$, and $v \equiv a_{1} \cdots a_{i-1} a_{i+2} \cdots a_{n}$. Hence,

$$
\begin{aligned}
u^{-1} & \equiv \bar{a}_{n} \cdots \bar{a}_{i+2} \bar{a}_{i+1} \bar{a}_{i} \bar{a}_{i-1} \cdots \bar{a}_{1} \\
& \equiv \bar{a}_{n} \cdots \bar{a}_{i+2} \bar{a}_{i+1} a_{i+1} \bar{a}_{i-1} \cdots \bar{a}_{1} \underset{T_{1}}{\stackrel{1}{T_{n}}} \bar{a}_{n} \cdots \bar{a}_{i+2} \bar{a}_{i-1} \cdots \bar{a}_{1} \\
& \equiv v^{-1} .
\end{aligned}
$$

If $n=m+1$, then there is some $i \in\{1, \ldots, n-1\}$ such that $\left(a_{i} a_{i+1}, b_{i}\right) \in T_{1}$, and $v \equiv a_{1} \cdots a_{i-1} b_{i} a_{i+2} \cdots a_{n}$. But $\left(a_{i} a_{i+1}, b_{i}\right) \in T_{1}$ implies $\left(\bar{a}_{i+1} \bar{a}_{i}, \bar{b}_{i}\right) \in T_{1}$, since $\Sigma_{1}^{*} / \stackrel{\leftrightarrow}{\leftrightarrow}$ is a group. Hence,

$$
u^{-1} \equiv \bar{a}_{n} \cdots \bar{a}_{i+2} \bar{a}_{i+1} \bar{a}_{i} \bar{a}_{i-1} \cdots \bar{a}_{1} \stackrel{1}{T_{1}} \bar{a}_{n} \cdots \bar{a}_{i+2} \bar{b}_{i} \bar{a}_{i-1} \cdots \bar{a}_{1} \equiv v^{-1} .
$$


$k \rightarrow k+1:$ If $u \underset{T_{1}}{\stackrel{k+1}{\rightarrow}} v$, then there is some $w \in \Sigma_{1}^{*}$ such that $u \underset{T_{1}}{\stackrel{k}{\rightarrow}} w \underset{T_{1}}{\stackrel{1}{\rightarrow}} v$. Now the induction assumption and the case $k=1$ imply $u^{-1} \underset{T_{1}}{\stackrel{k}{\rightarrow}} w^{-1} \underset{T_{1}}{\stackrel{1}{\rightarrow}} v^{-1}$, i.e., $u^{-1} \underset{T_{1}}{\stackrel{k+1}{\rightarrow}} v^{-1}$.

Now we can start to investigate the relation between $\varphi(a b)$ and $\varphi(c)$ for rules $(a b, c) \in T$. Several cases have to be distinguished depending on which of the letters $a, b, c$ are in $\Sigma_{1}$ and which are not. For $w \in \Sigma^{*}$, let $|w|_{\Sigma_{1}}:=\Sigma_{a \in \Sigma_{1}}|w|_{a}$, i.e., $|w|_{\Sigma_{1}}$ is the number of occurrences of letters from $\Sigma_{1}$ in $w$.

LEMMA 3.12. Let $(a b, c) \in T$ with $|a b c|_{\Sigma_{1}} \geqslant 2$. Then $\varphi(a b) \underset{T_{1}}{\stackrel{*}{\rightarrow}} \varphi(c)$.

Proof. (i) If $a, b, c \in \Sigma_{1}$, then $(a b, c) \in T_{1}$, and so $\varphi(a b) \equiv a b \underset{T_{1}}{\rightarrow} c \equiv \varphi(c)$.

(ii) If $a, b \in \Sigma_{1}$, and $c \in \Sigma-\Sigma_{1}$, then $c \in \Sigma_{2}$, and $v_{c} \equiv a b$ according to Corollary 3.6. Thus, $\varphi(a b) \equiv a b \equiv \varphi(c)$.

(iii) If $a, c \in \Sigma_{1}$, and $b \in \Sigma-\Sigma_{1}$, then $(a b, c) \in T$ implies $(\bar{a} c, b) \in T$. From (ii) we conclude that $\varphi(b) \equiv \bar{a} c$. Hence, $\varphi(a b) \equiv a \bar{a} c \rightarrow c \equiv \varphi(c)$.

(iv) If $b, c \in \Sigma_{1}$, and $a \in \Sigma-\Sigma_{1}$, then $(a b, c) \in T$ implies $(c \bar{b}, a) \in T$. Hence, $\varphi(a) \equiv c \bar{b}$, and so $\varphi(a b) \equiv c \bar{b} b \underset{T_{1}}{\rightarrow} c \equiv \varphi(c)$.

Lemma 3.13. Let $(a b, c) \in T$ with $a \in \Sigma_{i}, b \in \Sigma_{1}$, and $c \in \Sigma_{j}$ for some $i, j \geqslant 2$. Then $|i-j| \leqslant 1$, and $\varphi(a b) \underset{T_{1}}{\stackrel{*}{\rightarrow}} \varphi(c)$.

Proof. Since $a \in \Sigma_{i}$, we have $a^{-1} \equiv a_{1} \cdots a_{i} \in \Sigma_{1}^{*}$, and $\varphi(a) \equiv v_{a} \equiv \bar{a}_{i} \cdots \bar{a}_{1}$, and $c \in \Sigma_{j}$ implies $c^{-1} \equiv c_{1} \cdots c_{j} \in \Sigma_{1}^{*}$, and $\varphi(c) \equiv v_{c} \equiv \bar{c}_{j} \cdots \bar{c}_{1}$.

Since $\Sigma^{*} / \underset{T}{\leftrightarrow}$ is a group, $a b \underset{T}{\leftrightarrow} c$ implies $\bar{b} a_{1} \cdots a_{i} \equiv(a b)^{-1} \underset{T}{\stackrel{*}{\leftrightarrow}} c^{-1} \equiv c_{1} \cdots c_{j}$, and $a_{1} \cdots a_{i} \equiv a^{-1} \stackrel{*}{\leftrightarrow} b c_{1} \cdots c_{j}$. Now $c \in \Sigma_{j}$, and hence $j \leqslant i+1$. On the other hand, $a \in \Sigma_{i}$ implying $i \leqslant j+1$. Thus, $|i-j| \leqslant 1$. Now, three cases must be distinguished.

(i) $j=i-1:(a b, c) \in T$ and $b \in \Sigma_{1}$ imply $(c \bar{b}, a) \in T$. Hence,

$$
a_{1} \cdots a_{i} \equiv a^{-1} \stackrel{*}{\leftrightarrow}(c \bar{b})^{-1} \equiv b c_{1} \cdots c_{i-1} .
$$

But $a_{1} \cdots a_{i}$ is irreducible, and so $a_{1} \cdots a_{i} \equiv b c_{1} \cdots c_{i-1}$. Thus,

$$
\begin{aligned}
\varphi(a b) & \equiv \varphi(a) \varphi(b) \\
& \equiv \bar{a}_{i} \cdots \bar{a}_{1} b \\
& \equiv \bar{c}_{i-1} \cdots \bar{c}_{1} \bar{b} b{\overrightarrow{T_{1}}}_{\bar{c}_{i-1}} \cdots \bar{c}_{1} \\
& \equiv \varphi(c) .
\end{aligned}
$$

(ii) $j=i:(a b, c) \in T$ implies that

$$
\bar{b} a_{1} \cdots a_{i} \equiv(a b)^{-1} \stackrel{*}{\leftrightarrow} c^{-1} \equiv c_{1} \cdots c_{i} .
$$

Since $c_{1} \cdots c_{i}$ as well as $a_{1} \cdots a_{i}$ are irreducible, this means that $\left(\bar{b} a_{1}, c_{1}\right) \in T$ and $a_{\lambda} \equiv c_{\lambda}$ for $\lambda=2, \ldots, i$. Hence, $\left(\bar{b} a_{1}, c_{1}\right) \in T_{1}$, and so $\left(\bar{a}_{1} b, \bar{c}_{1}\right) \in T_{1}$. Thus,

$$
\varphi(a b) \equiv \bar{a}_{i} \cdots \bar{a}_{1} b \underset{T_{1}}{\rightarrow} \bar{a}_{i} \cdots \bar{a}_{2} \bar{c}_{1} \equiv \bar{c}_{i} \cdots \bar{c}_{1} \equiv \varphi(c) \text {. }
$$


(iii) $j=i+1:(a b, c) \in T$ implies that

$$
\bar{b} a_{1} \cdots a_{i} \equiv(a b)^{-1} \underset{T}{\leftrightarrow} c^{-1} \equiv c_{1} \cdots c_{i+1} .
$$

Since $c_{1} \cdots c_{i+1}$ is irreducible, we have $\bar{b} a_{1} \cdots a_{i} \equiv c_{1} \cdots c_{i+1}$. Hence,

$$
\varphi(a b) \equiv \bar{a}_{i} \cdots \bar{a}_{1} b \equiv \bar{c}_{i+1} \cdots \bar{c}_{1} \equiv \varphi(c) .
$$

A symmetric lemma can be proved accordingly.

LemmA 3.14. Let $(a b, c) \in T$ with $a \in \Sigma_{1}, b \in \Sigma_{i}$, and $c \in \Sigma_{j}$ for some $i, j \geqslant 2$. Then $|i-j| \leqslant 1$, and $\varphi(a b) \underset{T_{1}}{\stackrel{*}{\rightarrow}} \varphi(c)$.

It remains to consider those rules $(a b, c) \in T$ for which $a$ and $b$ both are in $\Sigma-\Sigma_{1}$. To handle these rules, we need

LEMMA 3.15. Let $u, v \in \Sigma_{1}^{*}$ be irreducible modulo $T$, and let $c, d \in \Sigma_{1}$ such that $u d v \underset{T}{\stackrel{*}{\rightarrow}} c$. Then $u d v \underset{T_{1}}{\stackrel{*}{\rightarrow}} c$.

Proof. Since $u$ and $v$ are irreducible modulo $T$, the reduction $u d v \underset{T}{*} c$ is of the following form:

$$
u d v \equiv u_{0} d_{0} v_{0} \underset{T}{\rightarrow} u_{1} d_{1} v_{1} \underset{T}{\rightarrow} \cdots \underset{T}{\rightarrow} u_{k} d_{k} v_{k} \underset{T}{\rightarrow} c
$$

where in each step the words $u_{i}, v_{i} \in \Sigma_{1}^{*}$ are irreducible, and one of the following three conditions is met:

(i) $u_{i+1} \equiv u_{i}, v_{i} \equiv f_{i} v_{i+1}$ with $f_{i} \in \Sigma_{1}$, and $\left(d_{i} f_{i}, d_{i+1}\right) \in T$ with $d_{i+1} \in \Sigma$,

(ii) $v_{i+1} \equiv v_{i}, u_{i} \equiv u_{i+1} g_{i}$ with $g_{i} \in \Sigma_{1}$, and $\left(g_{i} d_{i}, d_{i+1}\right) \in T$ with $d_{i+1} \in \Sigma$,

(iii) $u_{i} \equiv u_{i+1} g_{i}, v_{i} \equiv f_{i} v_{i+1}$ with $g_{i}, f_{i} \in \Sigma_{1}$, and $\left(\left(g_{i} d_{i}, 1\right) \in T\right.$ and $d_{i+1} \equiv f_{i}$ or $\left(d_{i} f_{i}, 1\right) \in T$ and $\left.d_{i+1} \equiv g_{i}\right)$.

Thus, in each step the rule applied is of the form $(a b, c)$ with $a \in \Sigma_{1}$ or $b \in \Sigma_{1}$. Hence, $\varphi(a b) \stackrel{*}{\rightarrow} \varphi(c)$ for every rule applied during this reduction according to Lemmas 3.12-3.14. Therefore, we have for each $i=0, \ldots, k-1$ :

$$
\begin{aligned}
\varphi\left(u_{i} d_{i} v_{i}\right) & \equiv u_{i} \varphi\left(d_{i}\right) v_{i} \stackrel{*}{\rightarrow} u_{i+1} \varphi\left(d_{i+1}\right) v_{i+1} \\
& \equiv \varphi\left(u_{i+1} d_{i+1} v_{i+1}\right)
\end{aligned}
$$

and so

$$
u d v \equiv \varphi\left(u_{0} d_{0} v_{0}\right) \underset{T_{1}}{\stackrel{*}{\rightarrow}} \varphi(c) \equiv c .
$$

Now we are ready to deal with the remaining rules that are of the form $(a b, c)$ with $a \in \Sigma_{i}$, and $b \in \Sigma_{j}$ for some $i, j \geqslant 2$.

LEMMA 3.16. Let $(a b, c) \in T$ with $a \in \Sigma_{i}, b \in \Sigma_{j}$ with $i, j \geqslant 2$, and $c \in \Sigma_{1}$. Then $\varphi(a b) \underset{T_{1}}{\stackrel{*}{\rightarrow}} \varphi(c)$.

Proof. Since $a \in \Sigma_{i}$, we have $a^{-1} \equiv a_{1} \cdots a_{i} \in \Sigma_{1}^{*}$, and $\varphi(a) \equiv \bar{a}_{i} \cdots \bar{a}_{1}$. Since $b \in \Sigma_{j}, b^{-1} \equiv b_{1} \cdots b_{j}$, and $\varphi(b) \equiv \bar{b}_{j} \cdots \bar{b}_{1}$.

$$
(a b)^{-1} \equiv b_{1} \cdots b_{j} a_{1} \cdots a_{i} \underset{T}{*} \bar{c} .
$$


But $b_{1} \cdots b_{j}$ and $a_{1} \cdots a_{i}$ are irreducible. Hence $b_{1} \cdots b_{j} a_{1} \cdots a_{i} \underset{T_{1}}{\stackrel{*}{c}} \bar{c}$ according to the previous lemma. Thus,

$$
\begin{aligned}
\varphi(a b) & \equiv \bar{a}_{i} \cdots \bar{a}_{1} \bar{b}_{j} \cdots \bar{b}_{1} \\
& \equiv\left(b_{1} \cdots b_{j} a_{1} \cdots a_{i}\right)^{-1} \underset{T_{1}}{\stackrel{*}{\longrightarrow}}(\bar{c})^{-1} \\
& \equiv c \\
& \equiv \varphi(c)
\end{aligned}
$$

according to Lemma 3.11 .

Thus, we have shown so far that $\varphi(a b) \underset{T_{1}}{\stackrel{*}{\rightarrow}} \varphi(c)$ for all rules $(a b, c) \in T$ with $|a b c|_{\Sigma_{1}} \geqslant 1$. Hence, the proof of Theorem 3.2 is completed by

Lemma 3.17. Let $(a b, c) \in T$ with $a \in \Sigma_{i}, b \in \Sigma_{j}$, and $c \in \Sigma_{k}$ for some $i, j, k \geqslant 2$. Then $\varphi(a b) \underset{T_{1}}{\stackrel{*}{\rightarrow}} \varphi(c)$.

Proof. Let $a^{-1} \equiv a_{1} \cdots a_{i} \in \Sigma_{1}^{*}, b^{-1} \equiv b_{1} \cdots b_{j} \in \Sigma_{1}^{*}$, and $c^{-1} \equiv c_{1} \cdots c_{k} \in$ $\Sigma_{1}^{*}$. Now $(a b, c) \in T$ implies that

$$
b_{1} \cdots b_{j} a_{1} \cdots a_{i} \equiv(a b)^{-1} \stackrel{*}{\leftrightarrow} c^{-1} \equiv c_{1} \cdots c_{k} .
$$

The word $c_{1} \cdots c_{k}$ is irreducible, and so $b_{1} \cdots b_{j} a_{1} \cdots a_{i} \stackrel{*}{\rightarrow} c_{1} \cdots c_{k}$. But $b_{1} \cdots b_{j}$ and $a_{1} \cdots a_{i}$ are irreducible as well. Hence there are $l$ and $m$ such that $b_{1} \cdots b_{l-1} \equiv c_{1} \cdots c_{l-1}, b_{l} \cdots b_{j} a_{1} \cdots a_{m} \stackrel{*}{\rightarrow} c_{l}$, and $a_{m+1} \cdots a_{i} \equiv c_{l+1} \cdots c_{k}$. Now $c_{l} \in \Sigma_{1}$, and $b_{l} \cdots b_{j}$ as well as $a_{1} \cdots a_{m}$ are irreducible. Thus, Lemma 3.15 applies giving $b_{l} \cdots b_{j} a_{1} \cdots a_{m} \underset{T_{1}}{\stackrel{*}{\rightarrow}} c_{l}$, and so $b_{1} \cdots b_{j} a_{1} \cdots a_{i} \underset{T_{1}}{\stackrel{*}{*}} c_{1} \cdots c_{k}$. Hence,

$$
\begin{aligned}
\varphi(a b) & \equiv \bar{a}_{i} \cdots \bar{a}_{1} \bar{b}_{j} \cdots \bar{b}_{1} \\
& \equiv\left(b_{1} \cdots b_{j} a_{1} \cdots a_{i}\right)^{-1} \underset{T_{1}}{\stackrel{*}{\rightarrow}}\left(c_{1} \cdots c_{k}\right)^{-1} \\
& \equiv \bar{c}_{k} \cdots \bar{c}_{1} \\
& \equiv \varphi(c) . \quad
\end{aligned}
$$

Together Lemmas 3.11-3.17 imply

LEMMA 3.18. The mapping $\varphi$ defines an isomorphism from the group $\Sigma^{*} / \stackrel{\leftrightarrow}{T}$ onto the group $\Sigma_{1}^{*} / \stackrel{\leftrightarrow}{T_{1}}$.

This proves Theorem 3.2.

4. Concluding remarks. Theorem 3.2 may be used to prove a lot of algebraic and algorithmic properties for the class of groups presented by two-monadic reduced Church-Rosser Thue systems. For instance, by Kurosch's Theorem $[16,18]$ this class is subgroup-closed, i.e., finitely generated subgroups are also presented by two- 
monadic reduced Church-Rosser Thue systems. In addition, centralizers of elements are cyclic or finite groups.

Free products of a finitely generated free group with finitely many finite groups have nice algorithmic properties, i.e., the conjugacy problem, the root problem, the power problem, the order problem, and other problems are easily solvable. At first sight it is not clear at all how to solve these problems without using the characterization theorem, i.e., by using only properties of monadic Church-Rosser Thue systems. Nevertheless, Theorem 2.3 is effective in the following sense: Given $M_{0}$ it is possible to effectively construct a presentation for $G$ as a free product of a finitely generated free group with finitely many finite groups. Since $M_{0}$ is effectively constructible we may construct a presentation for $G$ as a free product from a given monadic presentation for $G$ and then apply the usual algorithms in free products for solving problems like the conjugacy problem.

Notice that the type of presentations considered here leads to efficient algorithms for solving the word problem, but this may happen at the expense of the size of the presentation: For finite groups a presentation by a reduced monadic Church-Rosser Thue system providing inverses of length one for the generators is actually the multiplication table representation.

It is conjectured that the class of groups which can be presented by monadic Church-Rosser Thue systems is exactly the class considered in this paper. The question arises of whether this same proof technique can be applied in the general case. Our first observation is that such Thue systems do exist.

ExAmple. Take $\Sigma=\{a, b, c, d\}$, and $T=\{(a b c, 1),(c a, d),(d b, 1),(b d, 1)\}$. Then $T$ is monadic, reduced, and Church-Rosser. Obviously, the generators $b$ and $d$ are invertible. Further, $a b c \underset{T}{\rightarrow} 1$, and $b c a_{\vec{T}} \rightarrow \vec{T} b \underset{T}{\rightarrow}$, i.e., $a$ is invertible, and cap $\underset{T}{\rightarrow} d b \underset{T}{\rightarrow}$ 1, i.e., $c$ is also invertible. Hence, $\Sigma^{*} / \stackrel{\leftrightarrow}{\leftrightarrow}$ is a group.

Can the technique used to prove Theorem 3.2 be applied to Thue systems of this form? One difficulty consists in determining the generators that shall be kept.

ExAMPle (CONTINUED). $\Sigma_{1}=\{b, d\}$, and $\Sigma_{2}=\{a, c\}$. Now $\Sigma_{1}^{*} \cap \operatorname{IRR}(T)=$ $\left\{b^{i} \mid i \geqslant 0\right\} \cup\left\{d^{i} \mid i \geqslant 1\right\}$. Further, each $w \in \Sigma_{1}^{*}$ can be reduced to an irreducible $\hat{w} \in \Sigma_{1}^{*}$. Thus, there is no $w \in \Sigma_{1}^{*}$ such that $a \stackrel{\leftrightarrow}{\leftrightarrow} w$ or $c \stackrel{*}{\leftrightarrow} w$, i.e., the generators from $\Sigma_{2}$ cannot be expressed as words over $\Sigma_{1}$. And obviously, $\left(\Sigma_{1} ; T \cap\left(\Sigma_{1}^{*} \times \Sigma_{1}^{*}\right)\right)$ presents the group $F_{1}$, which is not isomorphic to the group $\Sigma^{*} / \stackrel{\leftrightarrow}{T} \cong F_{2}$, where $F_{i}$ is the free group on $i$ generators.

Thus, if this technique should work at all, then the partition of the set of generators $\Sigma$ must be chosen in a different way. One such way is the following.

Definition. Let $T$ be a finite, reduced, monadic, Church-Rosser Thue system over $\Sigma$ such that $\Sigma^{*} / \underset{T}{\stackrel{\leftrightarrow}{\leftrightarrow}}$ is a group, and $T$ contains rules with left-hand sides of length greater than two. For $a \in \Sigma$, define $i(a):=\min \left\{k \mid \exists u_{a} \in \Sigma^{*}: a \cdot u_{a} \underset{T}{\stackrel{k}{\rightarrow}} 1\right\}$. Take $\Sigma_{j}=\{a \in \Sigma \mid i(a)=j\}$.

Notice that for a Thue system the rules of which have left-hand sides of length two only, this new definition coincides with the one given in 3.4 . 
Since $\Sigma^{*} / \stackrel{\leftrightarrow}{\leftrightarrow}$ is a group, the Thue system $T$ contains rules of the form $(u, 1)$, and thus, $\Sigma_{1} \neq \varnothing$. Obviously, $\Sigma_{i} \cap \Sigma_{j}=\varnothing$ for $i \neq j$. Further, $\Sigma_{i+1} \neq \varnothing$ implies that $\Sigma_{i} \neq \varnothing$ as can be seen easily. Now we return to our example.

ExAmple (CONTINUED). $\Sigma=\{a, b, c, d\}$, and $T=\{(a b c, 1),(c a, d)$, $(d b, 1),(b d, 1)\}$. Hence $\Sigma_{1}=\{a, b, d\}$, and $\Sigma_{2}=\{c\}$.

Each word $w \in \Sigma_{1}^{*}$ can be reduced to an irreducible $\hat{w} \in \Sigma_{1}^{*}$. Thus, there is no $w \in \Sigma_{1}^{*}$ such that $c \underset{T}{\leftrightarrow} w$, i.e., again we cannot express the generators from $\Sigma_{2}$ as words over $\Sigma_{1}$.

So the question of whether the technique developed in $\$ 3$ can be applied to Thue systems that do have rules with left-hand sides of length greater than two remains open. If it applies, one has to find a different way of partitioning the given set of generators; if not, one has to find a new technique in which new letters are introduced to deal with Thue systems of this form that present groups.

\section{REFERENCES}

1. J. Avenhaus and K. Madlener, Subrekursive Komplexität bei Gruppen I. Gruppen mit vorgeschriebener Komplexität, Acta Inf. 9 (1977), 87-104.

2. On groups defined by monadic Thue systems, Algebra, Combinatorics, and Logic in Computer Science, Colloq. Math. Soc. János Bolyai, vol. 42, 1983, pp. 63-71.

3. J. Avenhaus, R. Book and C. Squier, On expressing commutativity by finite Church-Rosser presentations: a note on commutative monoids, RAIRO Inform. Théor. 18 (1984), 47-52.

4. R. Book, Confluent and other types of Thue systems, J. Assoc. Comput. Mach. 29 (1982), 171-182.

5. The power of the Church-Rosser property in string rewriting systems, Proc. 6th Conf. on Automated Deduction, 1982, pp. 360-368.

6. When is a monoid a group? The Church-Rosser case is tractable, Theoret. Comput. Sci. 18 (1982), 325-331.

7. R. Book, M. Jantzen and C. Wrathall, Monadic Thue systems, Theoret. Comput. Sci. 19 (1982), $231-251$.

8. W. W. Boone, Certain simple unsolvable problems of group theory. I, II, III, IV, V, VI, Nederl. Akad. Wetensch. Proc. Ser. A 57 (1954), 231-237; 57 (1954), 492-497; 58 (1955), 252-256, 58 (1955); 571-577; 60 (1957), 22-27; 60 (1957), 227-232.

9. B. Buchberger and R. Loos, Algebraic simplification, Computer Algebra (Symbolic and Algebraic Computation), (B. Buchberger, G. E. Collins, and R. Loos, eds.), Computing Supplementum 4, SpringerVerlag, Wien/New York, 1982.

10. F. B. Cannonito, Hierarchies of computable groups and the word problem, J. Symbolic Logic 31 (1966), 376-392.

11. Y. Cochet, Church-Rosser congruences on free semigroups, Algebraic Theory of Semigroups, Colloq. Math. Soc. János Bolyai, North-Holland, Amsterdam, vol. 20, 1976, pp. 51-60.

12. M. Dehn, Uber unendliche diskontinuierliche Gruppen, Math. Ann. 71 (1911), 116-144.

13. R. H. Haring-Smith, Groups and simple languages, Trans. Amer. Math. Soc. 279 (1983), 337-356.

14. D. Kapur and P. Narendran, The Knuth-Bendix completion procedure and Thue systems, Proc. 3rd Conf. Foundations of Computer Science and Software Engineering (Bangalore, India, 1983).

15. G. Lallement, Semigroups and combinatorial applications, Wiley-Interscience, New York, 1979.

16. R. Lyndon and P. Schupp, Combinatorial group theory, Springer-Verlag, New York, 1977.

17. W. Magnus, Das Identitätsproblem für Gruppen mit einer definierenden Relation, Math. Ann. 106 (1932), 295-307.

18. W. Magnus, A. Karrass and D. Solitar, Combinatorial group theory, 2nd rev. ed., Dover, New York, 1976.

19. D. E. Muller and P. E. Schupp, Groups, the theory of ends, and context-free languages, J. Comput. System Sci. 26 (1983), 295-310. 
20. P. S. Novikov, On the algorithmic unsolvability of the word problem in group theory, Trudy Mat. Inst. Steklov 44 (1955).

21. F. Otto, Conjugacy in monoids with a special Church-Rosser presentation is decidable, Semigroup Forum 29 (1984), 223-240.

22. M. O. Rabin, Recursive unsolvability of group theoretic problems, Ann. of Math. (2) 67 (1958), 172-194.

23. J. R. Stallings, Group theory and three-dimensional manifolds, Yale Univ. Press, New Haven, Conn., 1971.

Fachbereich Informatik, Universität Kaiserslautern, Postfach 3049, 6750 Kaiserslautern, WeSt Germany (Current address of J. Avenhaus and K. Madlener)

Current address (F. Otto): Department of Computer Science, State University of New York, Albany, New York 12222 